\title{
Electroluminescence From the Ge Quantum Dot MOS Tunneling Diodes
}

\author{
M. H. Liao, C.-Y. Yu, T.-H. Guo, C.-H. Lin, and C. W. Liu, Senior Member, IEEE
}

\begin{abstract}
A Ge quantum dot (QD) light-emitting diode (LED) is demonstrated using a MOS tunneling structure for the first time. The oxide film was grown by liquid phase deposition at $50{ }^{\circ} \mathrm{C}$ to reduce the thermal budget. The infrared emission of $\sim 1.5 \mu \mathrm{m}$ was observed from Ge QD MOS LEDs, similar to the p-type-intrinsic-n-type structure reported previously. At the negative gate bias, the electrons in the Al gate electrode tunnel to the Ge QD through the ultrathin oxide and recombine radiatively with holes to emit the $\sim 1.5-\mu \mathrm{m}$ infrared. The electrons also recombine with holes in the $\mathrm{Si}$ cap, and the band edge emission from $\mathrm{Si}$ is also observed.
\end{abstract}

Index Terms-Ge quantum dot (QD) light-emitting diodes (LEDs), MOS tunneling diode.

\section{INTRODUCTION}

$\mathbf{I}$ T IS a long-sought goal to integrate ultralarge-scale integrated (ULSI) circuits with electrooptics to possibly overcome the speed limitation of electrical interconnects and to add extra functionalities on Si chip. Light-emitting diodes (LEDs) and detectors are essential devices to achieve this goal. The Si MOS LED has been demonstrated [1] to emit the 1.1- $\mu \mathrm{m}$ infrared at the $\mathrm{Si}$ band edge. The addition of Ge quantum dot (QD) into Si can tune the optical characteristic to longer wavelength, and the Ge QD MOS detector [2], [3] was reported to detect $1.3-$ and $1.5-\mu \mathrm{m}$ infrared signals. The $\sim 1.5-\mu \mathrm{m}$ infrared light emission was also observed in a previous study of electroluminescence (EL) from the Ge QD p-type-intrinsicn-type (p-i-n) diodes [4]. However, the p-i-n structure is not fully compatible with the ULSI process. The building device in ULSI is the MOS structure. In this letter, we report that the Ge QD MOS tunneling diode can emit both the $\sim 1.5-\mu \mathrm{m}$ infrared from the Ge QD and the $\sim 1.1-\mu \mathrm{m}$ infrared from $\mathrm{Si}$. The MOS tunneling diode reported here has the same structure used in the ULSI circuits, whereas the oxide is thin enough to have significant tunneling current from the metal to the

Manuscript received December 2, 2005; revised January 16, 2006. This work was supported by the National Science Council of Taiwan, R.O.C. under Contracts 93-2215-E-002-003, 93-2215-E-002-017, 94-2215-E-002-040, and 94-2215-E-002-041, and the Asian Office of Aerospace Research and Development (AOARD), U.S. Airforce. The review of this letter was arranged by Editor P. Yu.

M. H. Liao and T.-H. Guo are with the Department of Electrical Engineering and the Graduate Institute of Electro-Optical Engineering, National Taiwan University, Taipei 106, Taiwan, R.O.C.

C.-Y. Yu and C.-H. Lin are with the Department of Electrical Engineering and the Graduate Institute of Electronics Engineering, National Taiwan University, Taipei 106, Taiwan, R.O.C. (e-mail: chee@cc.ee.ntu.edu.tw).

C. W. Liu is with the Department of Electrical Engineering and the Graduate Institute of Electro-Optical Engineering and the Department of Electrical Engineering and the Graduate Institute of Electronics Engineering, National Taiwan University, Taipei 106, Taiwan, R.O.C.

Digital Object Identifier 10.1109/LED.2006.870416

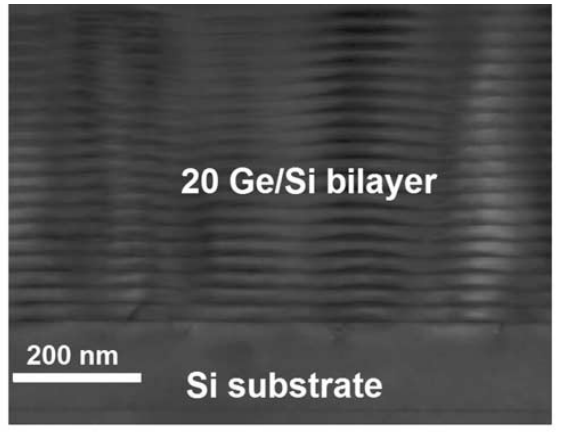

(a)

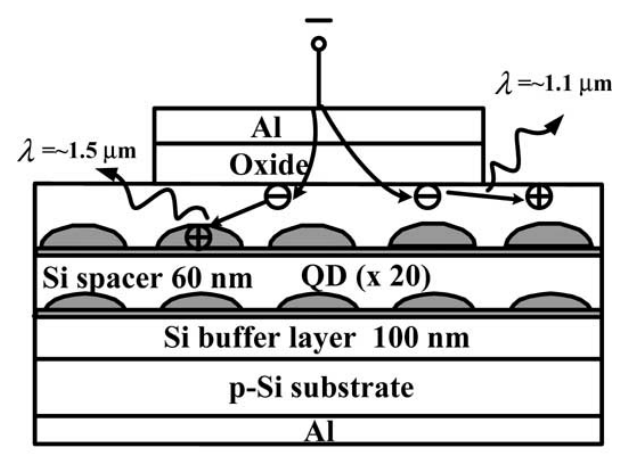

(b)

Fig. 1. (a) Cross-sectional TEM micrograph of the self-assembled Ge QD structure. (b) Schematic diagram of the Ge QD MOS LED device structure.

substrate. The fabrication for MOS LED does not need the n-type doping in the ultrahigh-vacuum chemical vapor deposition (UHV/CVD).

\section{DeVICE FABRicAtion}

The $\mathrm{Si} / \mathrm{Ge}$ QDs are prepared by UHV/CVD on p-type $\mathrm{Si}$ (001) substrates with resistivities in the range of $15-25 \Omega \cdot \mathrm{cm}$. After an Si buffer layer of $100 \mathrm{~nm}$ was grown, 20 periods of Ge QD structure with base and height of $\sim 120$ and $\sim 7 \mathrm{~nm}$, respectively, were grown at a temperature of $600{ }^{\circ} \mathrm{C}$ under the Stranski-Krastanov (SK) growth mode [5] as shown in Fig. 1(a). The Ge layers are separated by $60-\mathrm{nm} \mathrm{Si} \mathrm{spacer}$ layers. The Ge dot fabricated by the SK growth mode can lead to an increase of the luminescence efficiency because of the localization of excitons in the dots [6] and the increase of hole concentration in the dots. The area density of Ge islands is about $4 \times 10^{9} \mathrm{~cm}^{-2}$. A 3-nm (nominal thickness) Si cap was deposited above the top layer of the self-assembled Ge layer as a cap layer for the subsequent deposition of liquid phase deposition (LPD) oxide. The undoped $\mathrm{Si} / \mathrm{Ge}$ layers has a background hole concentration of $\sim 1 \times 10^{16} \mathrm{~cm}^{-3}$. $\mathrm{Al}$ is 


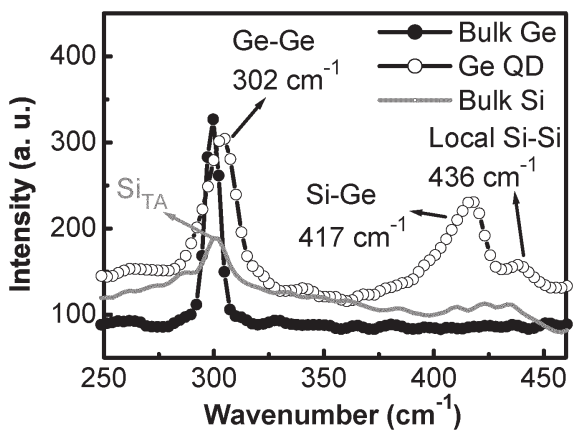

Fig. 2. Raman spectra of bulk $\mathrm{Si}$, bulk $\mathrm{Ge}$, and $\mathrm{Ge} \mathrm{QD}$. Ge/Ge, $\mathrm{Si} / \mathrm{Ge}$, and local Si/Si optical modes can be found at around 302, 417, and $436 \mathrm{~cm}^{-1}$, respectively.

used for the gate electrode and the back contact. Fig. 1(b) shows the device structure. To avoid material degradation such as strain relaxation and Ge out diffusion, the low-temperature oxide (LPD oxide) is used to reduce the thermal budget of the $\mathrm{Si} / \mathrm{Ge}$ device process [3]. The tunneling current of electrons at the negative bias can cause electron-hole recombination at the oxide/Si interface and in the Ge QDs to emit $\sim 1.1$ - and $\sim 1.5-\mu \mathrm{m}$ infrared, respectively. The momentum conservation required by the indirect band gap can be achieved by Si/oxide roughness scattering, localized holes, and phonons scattering. Details were given in [7] and [8].

\section{RESUlTS AND DISCUSSION}

Fig. 2 shows Raman spectra excited by the 514-nm Ar laser for the Ge QD, bulk Ge, and bulk Si. In addition to the strong $\mathrm{Si}$ substrate signal at $520 \mathrm{~cm}^{-1}, \mathrm{Ge}-\mathrm{Ge}, \mathrm{Si}-\mathrm{Ge}$, and local Si-Si vibrational peaks were also observed at $\sim 302, \sim 417$, and $\sim 436 \mathrm{~cm}^{-1}$, respectively. The appearance of the Si-Ge and the local Si-Si vibrational peaks implies the formation of $\mathrm{Si}-\mathrm{Ge}$ alloy in the wetting layers [9]. On the other hand, the Ge-Ge peak is most possibly contributed by the islands [9]. As compared with the bulk Ge, the Raman shift of the $\mathrm{Ge} / \mathrm{Ge}$ phonon frequency of the Ge QD with respect to the bulk Ge peak can be determined $\left(\sim 2 \mathrm{~cm}^{-1}\right)$. The possible factors responsible for this frequency shift are the alloying of the Ge QDs with Si spacers [10], the phonon confinement effect in the QDs, and the effects of compressive strain [11]. The first two factors reduce the phonon frequency, but the last mechanism increases the phonon frequency. Energy dispersion spectrometry shows the Ge dots transform into $\mathrm{Si}_{0.46} \mathrm{Ge}_{0.54}$ dots due to $\mathrm{Si} / \mathrm{Ge}$ interdiffusion at the growth temperature of $600{ }^{\circ} \mathrm{C}$.

Fig. 3 shows the photoluminescence (PL) spectra at different temperatures from the Ge QD before the fabrication of the MOS LED device at a pump power of $60 \mathrm{~W} / \mathrm{cm}^{2}$. There are two PL peaks located at $\sim 1.1$ and $\sim 0.85 \mathrm{eV}$. These two peaks are most likely from the optical transitions related to the $\mathrm{Si}$ substrate and the Ge QD [12]. Fig. 4 shows the EL spectra at different temperatures from the Ge QD MOS LED samples with a device size of $4 \times 10^{-4} \mathrm{~cm}^{2}$. The drive current is $100 \mathrm{~mA}$ at a gate voltage of $-8 \mathrm{~V}$. Device temperatures of $\sim 340, \sim 250, \sim 160$, and $\sim 110 \mathrm{~K}$ are obtained from the fitting of the EL line shapes from the Si signal with the

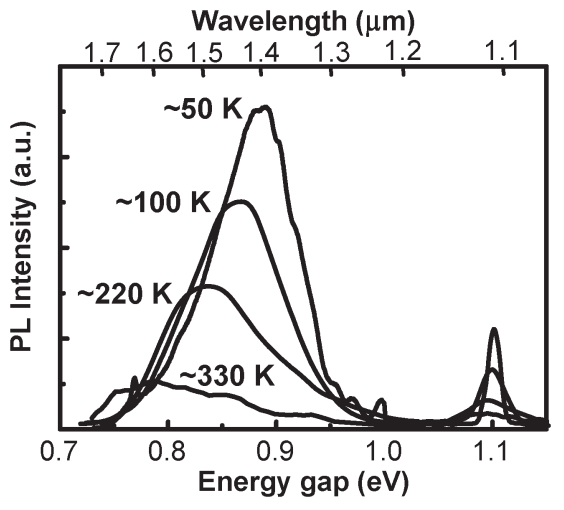

Fig. 3. PL spectra of the Ge QD at different temperatures. The infrared emission of $\sim 1.1$ - and $\sim 1.5 \mu \mathrm{m}$ was observed from the transition of $\mathrm{Si}$ and the Ge QD.

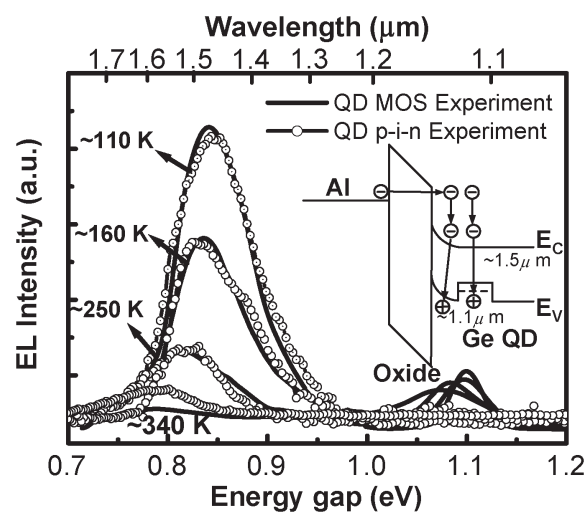

Fig. 4. EL spectra of the Ge QD MOS LED at different temperatures. The inset shows the energy band diagram of the Ge QD MOS LED at the accumulation bias. The EL spectra of the Ge QD p-i-n diode are also shown for reference.

electron-hole-plasma recombination model [1], [7], whereas the temperatures of the cold finger in the EL measurement are $300,200,100$, and $10 \mathrm{~K}$, respectively. The temperature difference is due to the device heating. Because our Ge dot has a wide base $(\sim 120 \mathrm{~nm})$ and a short height $(\sim 7 \mathrm{~nm})$, the QD can be approximated by the quantum well in the electron-hole-plasma recombination model [13]. The band diagram of the Ge QD MOS LED at the accumulation condition is shown in the inset of Fig. 4. The traps in the LPD oxide and the small thickness $(\sim 2 \mathrm{~nm})$ allow the significant tunneling electrons to inject from the $\mathrm{Al}$ electrode into the semiconductor. At a negative bias, the electrons tunnel from the $\mathrm{Al}$ gate to the substrate. Meanwhile, the negative gate bias also attracts holes in the p-type semiconductor and QDs, and the tunneling electrons can recombine with holes at the oxide/Si interface and in the Ge QD to emit $\sim 1.1$ - and $\sim 1.5-\mu \mathrm{m}$ infrared, respectively [Fig. 1(b)]. The EL spectra of similar Ge QD p-i-n diode are also shown for reference. The infrared emission of $\sim 1.5 \mu \mathrm{m}$ observed from the Ge QD MOS LED is similar to the p-i-n structure reported previously [4]. The external quantum efficiency for $\sim 1.5-\mu \mathrm{m}$ light emission at $\sim 110 \mathrm{~K}(\sim 340 \mathrm{~K})$ from the Ge MOS LED was observed to be $\sim 4 \times 10^{-5}\left(\sim 6 \times 10^{-6}\right)$, as compared to $\sim 9.5 \times 10^{-5}\left(\sim 1.2 \times 10^{-5}\right)$ for the Ge QD p-i-n diode. Note that in our quantum efficiency measurement, we cannot collect all the light from the device, and the number presented here 
is a lower bound of the true quantum efficiency. The efficiency is too low to have product applications, but the EL of the MOS structure is a good characterization tool for material quality and can be used in the in-line inspection in the industry. Note that the MOS structure is much simpler than the p-i-n structure to measure the EL spectra.

\section{CONCLUSION}

The $\sim 1.5-\mu \mathrm{m}$ Ge QD MOS LED, which is fully compatible with the ULSI process, is reported for the first time. The origin of the emission is due to the radiative recombination between the electrons and the holes confined in the Ge QD. The electrons also recombined with the holes at the Si/oxide interface, and the band edge light emission from $\mathrm{Si}$ is also observed.

\section{ACKNOWLEDGMENT}

The authors would like to thank Prof. S. T. Chang and Prof. C.-T. Chia for helpful discussion.

\section{REFERENCES}

[1] C. W. Liu, M. H. Lee, M.-J. Chen, I. C. Lin, and C.-F. Lin, "Roomtemperature electroluminescence from electron-hole plasmas in the metal oxide silicon tunneling diodes," Appl. Phys. Lett., vol. 76, no. 12, pp. 1516-1518, Mar. 2000.

[2] B.-C. Hsu, S. T. Chang, T. C. Chen, P.-S. Kuo, P. S. Chen, Z. Pei, and C. W. Liu, "A high efficient $820 \mathrm{~nm}$ MOS Ge quantum dot photodetector," IEEE Electron Device Lett., vol. 24, no. 5, pp. 318-320, May 2003.
[3] B.-C. Hsu, S. T. Chang, C.-R. Shie, C.-C. Lai, P. S. Chen, and C. W. Liu, "High efficient $820 \mathrm{~nm}$ MOS Ge quantum dot photodetectors for shortreach integrated optical receivers with 1300 and $1500 \mathrm{~nm}$ sensitivity," in IEDM Tech. Dig., San Francisco, CA, 2002, pp. 91-94.

[4] Y. H. Peng, C.-H. Hsu, C. H. Kuan, C. W. Liu, P. S. Chen, M.-J. Tsai, and Y. W. Suen, "The evolution of electroluminescence in Ge quantumdot diodes with the fold number," Appl. Phys. Lett., vol. 85, no. 25, pp. 6107-6109, Dec. 2004.

[5] T. I. Kamins, D. A. A. Ohlberg, R. S. Williams, W. Zhang, and S. Y. Chou, "Positioning of self-assembled, single-crystal, germanium islands by silicon nanoimprinting," Appl. Phys. Lett., vol. 74, no. 12, pp. 1773-1775, Mar. 1999.

[6] R. Apetz, L. Vescan, A. Hartman, C. Dieker, and H. Luth, "Photoluminescence and electroluminescence of SiGe dots fabricated by island growth," Appl. Phys. Lett., vol. 66, no. 4, pp. 445-447, Jan. 1995.

[7] C. W. Liu, M.-J. Chen, I. C. Lin, M. H. Lee, and C.-F. Lin, "Temperature dependence of the electron-hole-plasma electroluminescence from metal-oxide-silicon tunneling diodes," Appl. Phys. Lett., vol. 77, no. 8, pp. 1111-1113, Aug. 2000.

[8] C. W. Liu, M. H. Lee, M.-J. Chen, C.-F. Lin, and M. Y. Chern, "Roughness-enhanced electroluminescence from metal oxide silicon tunneling diodes," IEEE Electron Device Lett., vol. 21, no. 12, pp. 601-603, Dec. 2000.

[9] J. L. Liu, Y. S. Tang, K. L. Wang, T. Radetic, and R. Gronsky, "Raman scattering from a self-organized Ge dot superlattice," Appl. Phys. Lett., vol. 74, no. 13, pp. 1863-1865, Mar. 1999.

[10] W. J. Brya, "Raman scattering in Ge-Si alloys," Solid State Commun., vol. 12 , no. 4 , pp. $253-257$, Feb. 1973.

[11] F. Cerdeira, A. Pinczuk, J. C. Bean, B. Batlogg, and B. A. Wilson, "Raman scattering from $\mathrm{Ge}_{x} \mathrm{Si}_{1-x} / \mathrm{Si}$ strained-layer superlattices," Appl. Phys. Lett., vol. 45, no. 10, pp. 1138-1140, Nov. 1984.

[12] L. P. Rokhinson, D. C. Tsui, J. L. Benton, and Y.-H. Xie, "Infrared and photoluminescence spectroscopy of p-doped self-assembled Ge dots on Si," Appl. Phys. Lett., vol. 75, no. 16, pp. 2413-2415, Oct. 1999.

[13] K. Eberl, O. G. Schmidt, O. Kienzle, and F. Ernst, "Preparation and optical properties of Ge and C-induced Ge quantum dots on Si," Thin Solid Films, vol. 373 , no. $1-2$, pp. 164-169, Sep. 2000. 